\title{
LA PROMOCIÓN EMPRESARIAL DE LA MICRO EMPRESA EN EL AA.HH. PUERTO PACHACÚTEC DEL DISTRITO DE VENTANILLA, REGIÓN CALLAO
}

\author{
César Augusto Bellido Salcedo* \\ ca.bellido@yahoo.es \\ bellido.ca@pucp.edu.pe
}

\begin{abstract}
RESUMEN
Hoy en día nuestro país está viviendo una etapa de estabilidad económica, especialmente en los últimos 12 años con un crecimiento sostenido y sólido, pero el crecimiento se debe plasmar en desarrollo. Si bien es cierto que la pobreza ha disminuido, es importante que un estrato de la sociedad sea impulsado y promovido hacia una mayor inclusión económica. Los asentamientos humanos (AA. HH.), que son parte de esos estratos, presentar una oportunidad para plasmar la promoción de la generación de las micro empresas como formas de desarrollo y de formalidad.

El presente proyecto tiene como propósito analizar la generación de la promoción de micro negocios en el AA.HH. Puerto Pachacútec, del distrito de Ventanilla en la región Callao. Este estudio está enmarcado en un trabajo de campo y en un análisis sobre el estado actual y sobre las posibilidades que se pueden presentar para el desarrollo de este asentamiento humano mediante la generación de micro empresas.

En la primera etapa se ha elaborado una base de datos de la cantidad de comerciantes que en la actualidad están ejerciendo alguna actividad económica en dicha zona. Así mismo se ha hecho un estudio, en base a un muestreo de la población, sobre el nivel educativo, acceso a servicios públicos, medios de comunicación y percepción sobre el apoyo recibido del gobierno, tanto central como regional y local. Finalmente se analizan los resultados y se plantean propuestas para cada una de las variables presentadas, todas ellas enfocadas en el tema central de nuestro estudio, considerando el posible impacto social.
\end{abstract}

Palabras claves: Investigación, Sensibilización, Capacitación, Desarrollo, Emprendimiento

* Magíster en Administración de Negocios por la Pontificia Universidad Católica del Perú, Contador Público por la Universidad Ricardo Palma. Doctorando en Administración. Profesor universitario en pre grado y post grado en las áreas de Dirección Estratégica, Finanzas y Auditoría. Asesor y consultor de empresas. 


\begin{abstract}
Today, Peru is experiencing a period of economic stability. In the last 12 years, the country has had especially solid and sustainable growth. However, growth should translate into development. While it is true that poverty has declined, a stratum of society should be encouraged and promoted to greater economic inclusion. Human settlements (HHSS), which are part of these social stratums, provide an opportunity to capture the generation of micro-enterprises as forms of formality and development.

This project aims to analyze the generation of micro-businesses promotion in the in Puerto Pachacutec Human settlement, located in the district of Ventanilla in the Callao region. This project is framed in a field study, as an analysis of the current status and possibilities that may arise in the development of this human settlement.

In the first stage of the project, a database has been developed of the number of traders who are currently exerting an economic activity in the area. A study has also been done (based on a sampling of the population) of the educational level, accessibility to public services, social media engagement and perception of the support received from the government, both on central and local levels. Finally, we will analyze the results and consider proposals for each of the variables presented, all of them focused on the central theme of our study and to see results of the possible social impact.
\end{abstract}

Keywords: Research, Awareness, Training, Development, Entrepreneurship

\section{INTRODUCCIÓN}

El emprendimiento en la sociedad peruana se está viendo reflejado en el crecimiento que tienen las micro empresas. La misma necesidad de autoemplearse lleva a que las familias en distintas partes del país y también de distintas realidades vean a la micro empresa como una forma de desarrollo y, en algunos, casos de realización personal.

Los asentamientos humanos ubicados mayormente en las ciudades grandes serán el escenario en el que muchas familias harán frente a sus diversas necesidades realizando algún oficio o comercio, actividad con la cual podrán solventar los requerimientos propios del costo vida.

El fomento a la micro empresa en nuestro país se estableció en el año 2003 con la promulgación de la Ley $\mathrm{N}^{\circ} 28015$ "Ley de promoción y formalización de la micro y pequeña empresa”, creándose un régimen laboral especial de tipo temporal que buscaba fomentar la formalización y contratación de personal mediante la reducción de beneficios laborales. Desde ese entonces hasta la actualidad se han estado publicando normas complementarias que establecieron a las micro empresas como parte de una realidad en nuestro país.

El presente proyecto de investigación está dirigido al estudio de la promoción empresarial de la micro empresa en el AA.HH. Puerto Pachacútec del Distrito de Ventanilla, Región Callao. En el presente documento veremos cómo en esta comunidad se desarrollaron las micro empresas y ver cómo los pobladores ponen en marcha estos negocios como medio de desarrollo económico.

También se presentarán los resultados de trabajos del trabajo de campo que irán acompañados de un análisis de interpretación y por último de la bibliografía utilizada como material de consulta.

Finalmente, agradezco a la Universidad Nacional Mayor de San Marcos (UNMSM) por la oportunidad de desarrollar este proyecto de investigación, y también a la estudiante universitaria Jimena Bellido Jara por el apoyo brindado.

\section{PLANTEAMIENTO DEL ESTUDIO}

1. El desarrollo económico de las sociedades nos lleva a tomar en cuenta la responsabilidad de las instituciones de formación académica y profesional como entes que generan el conocimiento evolutivo que busca una mejor calidad de vida de las personas. El emprendimiento constituye hoy en día la intensión sólida de las personas de desarrollar actividades que generen una mayor independencia económica y social.

2. En países como el nuestro, donde a pesar de los logros alcanzados todavía se cuenta con una población que requiere de oportunidades y de apoyo para su propio crecimiento, es importante desarrollar proyectos que vinculen a la población con la universidad en una misma intensión: el desarrollo de su comunidad y de las personas. 
3. Las micro empresas son el medio de desarrollo económico que mejor se ha podido plasmar en los asentamientos humanos del Perú ya que cuentan con normas cuyo propósito es el de promover la sostenibilidad de las actividades económicas en los niveles socio económicos C y D.

Nuestro propósito es promover los micro negocios en el AA.HH. Puerto Pachacútec, del distrito de Ventanilla en la región Callao, como una forma de desarrollo autogestionaria.

Nuestro proyecto abarcará:

- Conocimiento cultural de la población de un asentamiento humano.

- Identificación del potencial para las actividades productivas.

- Concientización de la población para una cultura de emprendimiento.

- Capacitación y asesoría en la formación de las actividades productivas de las micro empresas.

- Impacto social y económico.

El propósito del presente proyecto es el de generar en la población del AA.HH. Puerto Pachacútec, la creación de micro empresas, con el objetivo que esa zona pueda convertirse en un foco de desarrollo económico tanto en el ámbito productivo como artesanal.

La Facultad de Ciencias Administrativas de la UNMSM tiene, como parte de sus objetivos, la proyección social con la comunidad, por la cual nace un compromiso: el de contribuir al desarrollo de la sociedad.

\section{MARCO TEÓRICO Y DEFINICIÓN DE CONCEPTOS}

\section{Definición de las micro empresas}

Las micro empresas son unidades de producción económica que cuentan con menos de 10 empleados y generan ventas anuales menores a 150 Unidades Impositivas Tributarias (UIT).

En un contexto global esta definición comprende algunos factores. Una definición exacta de una micro empresa depende de la legislación de cada país. En la Unión Europea, y por tanto en todos los países que la conforman, se entiende por micro empresa a aquellas empresas que tienen, entre otros requisitos, 10 empleados o menos, una facturación menor o igual a $\propto 2$ millones y un volumen anual de activos igual o inferior a esa cantidad.[1] En algunos lugares también son llamadas small office, home office (pequeña oficina, oficina en casa) o SoHo. Empresas mayores, que no cuentan con este modelo de división del trabajo a menudo son llamadas pequeñas y medianas empresas. Las micro empresas surgen de un proyecto emprendedor por parte del dueño, quien a su vez suele ser el administrador de su propia empresa. Son negocios que venden sus productos en pequeñas cantidades y no requieren de gran inversión para su funcionamiento. Estas pequeñas empresas tienen la posibilidad de crecer y generar competitividad con sus similares (micro empresa n.d.).

Una definición de la micro empresa acorde a nuestra legislatura indica que “... es aquella unidad económica constituida por una persona natural o jurídica (por ejemplo EIRL. SRL. o SAC.) que tiene por objeto desarrollar actividades de extracción, transformación, producción, comercialización de bienes o prestación de servicios” (Matos Barzola, 2008).

Generalmente en este tipo de organizaciones, la producción y la administración son bastante simples; así mismo, están orientadas a la subsistencia y a generar autoempleo. Tal vez deberíamos comenzar por definir una micro empresa como aquella que es pequeña en todos aspectos: número de colaboradores, ventas, tecnología, sistemas de información, grado de especialización, e internacionalización o globalización (Alva Mercado, 1998).

Los siete principales rubros a los que se dedican son: comercios, restaurantes, talleres, construcción, confección, muebles y metal mecánica.

Las Micro empresas destacan por:

- Su empuje para sacar adelante el negocio

- Su ingenio para producir aun contando con recursos escasos

- Su bajo nivel de capacitación

- Crear empresas familiares cuyo objetivo es subsistir

- No distinguir entre cuentas de la empresa y del hogar 
La importancia de las Microempresas para la economía y la sociedad?

Las Microempresas (MES) son la principal fuente de empleo en el Perú: 96 de cada 100 empresas son de tamaño micro 7 de cada 10 trabajadores laboran en una de ellas.

Se estima que existen más de 5 millones de micro empresas, de las cuales más de 3.5 millones son informales; es decir, no están debidamente constituidas ante la ley ni pagan impuestos.

\section{Estado actual de las micro empresas}

A pesar de congregar al $70 \%$ de la población económicamente activa, las micro empresas solo generan el $30 \%$ de todo lo producido en el país. Ello refleja un bajísimo nivel de productividad, mismo que no representa ni la quinta parte de la del resto de empresas y se traduce en que sus niveles de ingreso son significativamente más bajos.

Los organismos internacionales, los gobiernos, el sector académico y las Organizaciones no gubernamentales canalizan recursos hacia programas de capacitación, brindan acceso al mercado, o ayuda financiera directa, orientadas a mejorar la competitividad y el nivel de vida de las micro empresas. Sin embargo, su apoyo aún es bastante limitado.

Por otra parte, las cajas regionales, municipales y distritales; las financieras; algunos bancos y algunas ONG compiten por ofrecer préstamos a las micro empresas, pero en cantidades restringidas.

\section{Propuesta de mejora de las micro empresas}

Una de las razones de la promoción de las micro empresas es plantear mecanismos para que dejen la informalidad, que en nuestro país está muy establecida. Ya Hernando de Soto mencionaba que "... la informalidad se produce cuando el Derecho impone reglas que exceden el marco normativo socialmente aceptado, no ampara las expectativas, elecciones y preferencias de quien no puede cumplir tales reglas y el Estado no tiene la capacidad coercitiva suficiente" (De Soto, año)

Para erradicar la pobreza y las abismales diferencias entre clases sociales, resulta indispensable capacitar y mejorar la competitividad de las micro empresas, integrándolas al resto de la economía. Entonces, para fortalecer el desarrollo de este sector en el Perú, es conveniente implementar iniciativas en los siguientes frentes (Campaña Nuñez, s. f.):
1. Responsabilidad Social: Tanto a nivel empresarial como individual, implica identificar y asumir activamente un rol de apoyo de cara a los que menos tienen. Por ejemplo, los alumnos de la Universidad del Pacífico, mediante el curso de Proyección Social, asesoran gratuitamente a micro empresas en materia financiera, contable, legal y estratégica para ayudar a fortalecer la competitividad de sus negocios.

2. Clustering: El desarrollo económico para las micro empresas requiere de un proceso de agrupamiento que les genere ahorros y les abra puertas a mayores mercados. El Estado está llamado a simplificar los trámites y requisitos que deben cumplir las micro empresas para agrupar agentes con características similares, con el objetivo de mejorar su eficiencia conjunta. Por su parte, las entidades financieras están llamadas a brindar mayor acceso al financiamiento, el cual a veces resulta imprescindible para que este tipo de empresa se consolide y crezca.

3. Linkaging: El problema no es ser pequeño sino estar aislado del resto de la economía. Tanto el sector privado como el sector público deben generar vínculos económicos, culturales y sociales con las micro empresas, con la finalidad de propiciar su integración. Por ejemplo, el $40 \%$ de las compras de bienes y servicios que realiza el Estado proviene de las micro empresas formales.

Hay mucho trabajo por realizar y es tarea de todos. Mediante iniciativas como las descritas se fortalecerá la inclusión social y mejorará la distribución de la riqueza, contribuyendo así con el desarrollo económico de todo el país.

\section{Clasificación de la micro empresa}

Tal como se citó, la definición de microempresa se basa de acuerdo a la legislación de cada país, y en ese sentido es posible que podría aplicarse a los tipos de microempresas.

En el Panorama Laboral 2006 (OIT, 2006) se presenta una caracterización de las metodologías para la estimación de la informalidad según la XVII Conferencia Internacional de Estadísticas del Trabajo (CIET). ${ }^{6}$ Este enfoque reconoce la existencia dual de sistemas de producción y empleo en los países de la región, diferenciándose por los grados de organización y niveles de capital, productividad y tecnología con que se desarrolla el proceso productivo (ver Cuadro $\mathrm{N}^{\mathrm{O}}$ 1). Esta estimación es utilizada para categorizar la estructura del empleo en formal e informal. 
En el Perú la clasificación de las empresas peruanas toma como referencia el monto de sus ventas anuales $-\mathrm{y}$ ya no el número de sus trabajadores- según se contempla en la Ley de Impulso al Desarrollo Productivo y al Crecimiento Empresarial. El objetivo es el de facilitar la inversión, impulsar el desarrollo productivo y el crecimiento empresarial. Un punto importante es que las micro empresas tendrán un plazo de tres años desde su constitución para subsanar in- fracciones laborales y tributarias sin la aplicación de sanciones.

La norma, señala además, que las micro empresas que superen durante dos años las 150 UIT podrán conservar por un año adicional el régimen especial laboral, mientras que las pequeñas empresas que superen las 1.700 UIT por dos años podrán conservar el mismo régimen durante otros tres años.

\section{Cuadro $N^{\circledR} 1$ Clasificación de las micro empresas}

\begin{tabular}{|c|c|c|}
\hline $\begin{array}{l}\text { Caracterización de metodologías } \\
\text { para la estimación de la informali- } \\
\text { dad Características }\end{array}$ & $\begin{array}{l}\text { Programa Regional del Empleo para } \\
\text { América Latina y el Caribe (PREALC- } \\
\text { OIT) }\end{array}$ & $\begin{array}{l}\text { XVII Conferencia Internacional de Estadísticos del } \\
\text { Trabajo (CIET) (empleo informal) } 2003\end{array}$ \\
\hline Zona y rama & Urbano - no agrícola. & $\begin{array}{l}\text { Debería abarcar zonas urbanas y rurales. Los países } \\
\text { que excluyen actividades agrícolas del ámbito de sus } \\
\text { estadísticas sobre el sector informal deberían elaborar } \\
\text { definiciones. }\end{array}$ \\
\hline \multirow{7}{*}{ Situación en el empleo } & $\begin{array}{l}\text { Trabajadores independientes o por cuenta } \\
\text { propia (excluye a profesionales y técni- } \\
\text { cos). }\end{array}$ & $\begin{array}{l}\text { Trabajadores por cuenta propia dueños de sus propias } \\
\text { empresas del sector informal (incluyendo profesion- } \\
\text { ales y técnicos si no tienen condiciones de formalidad, } \\
\text { en especial registro, conforme a la legislación nacio- } \\
\text { nal). Pueden incluirse todos los trabajadores por cuen- } \\
\text { ta propia dueños de sus propias empresas informales } \\
\text { o solamente los que no están registrados. }\end{array}$ \\
\hline & $\begin{array}{l}\text { Empleadores o propietarios de empresas } \\
\text { de cinco o menos ocupados. }\end{array}$ & $\begin{array}{l}\text { Empleadores dueños de sus propias empresas del } \\
\text { sector informal en función de una o varias condicio- } \\
\text { nes: (i) el tamaño de las unidades es inferior a un nivel } \\
\text { determinado de empleo; y (ii) no están registradas o } \\
\text { no ocupan empleados registrados. }\end{array}$ \\
\hline & $\begin{array}{l}\text { Asalariados en empresas de cinco o me- } \\
\text { nos ocupados. }\end{array}$ & $\begin{array}{l}\text { Asalariados que tienen empleos informales, tanto si } \\
\text { están empleados por empresas del sector formal o por } \\
\text { empresas del sector informal }\end{array}$ \\
\hline & $\begin{array}{l}\text { Trabajadores familiares o auxiliares no re- } \\
\text { munerados. }\end{array}$ & $\begin{array}{l}\text { Trabajadores familiares o auxiliares, independiente- } \\
\text { mente de si trabajan en empresas del sector formal } \\
\text { o informal. }\end{array}$ \\
\hline & & $\begin{array}{l}\text { Miembros de cooperativas de productores que no es- } \\
\text { tán formalmente constituidas como entidades legales. }\end{array}$ \\
\hline & Servicio doméstico. & $\begin{array}{l}\text { Asalariados de los hogares que tienen empleos infor- } \\
\text { males. }\end{array}$ \\
\hline & & $\begin{array}{l}\text { Trabajadores en actividades no especializadas de } \\
\text { producción o bienes para consumo final de su propio } \\
\text { hogar. }\end{array}$ \\
\hline Unidad de observación & Las personas ocupadas. & Los empleos. \\
\hline
\end{tabular}

Fuente: OIT, con base en información del PREALC y de la XVII CIET 


\section{HIPÓTESIS DEMOSTRADA EN EL ESTUDIO}

Se acostumbra a llamar a la micro empresa como un sector de subsistencia, perteneciente al sector informal de la economía, por lo que en la concepción común son un estadío temporal en la inserción al sector formal y de grandes empresas. Diversos autores explican la creación y existencia de la micro empresa como resultado de la incapacidad de un país para ofrecer empleo formal y como un elemento de subsistencia (Ruiz, 2004).

Dentro del estudio realizado en el AA.HH. Puerto Pachacútec del Distrito de Ventanilla, Región Callao y sobre la materia expuesta, vemos que la promoción de la micro empresa en esta zona todavía no está debidamente establecida, esto de acuerdo a lo visto en los trabajos de campo, realizados mediante distintos métodos y técnicas empleadas. La presencia del Estado en lo referente a promoción a través de las diversas instituciones no es notoria. Se aprecia la presencia de una fundación de la iglesia católica que desarrolla actividades de enseñanza técnica. Una de las realidades vistas es el aún asistencialismo del que depende el asentamiento humano. La aislada y eventual presencia de empresas privadas en campañas sociales mediante donaciones en fechas especiales contribuye a ese asistencialismo. Se va a requerir que las organizaciones del gobierno central o regional, implementen los servicios básicos para la problación como luz, agua y desague. Se ha avanzado ya en el suministro de luz pero el sumnistro de agua es notoriamente crítico más aún siendo la zona donde el costo del agua (vía venta mediante cisternas) es el más alto de la capital. Este punto es una tarea pendiente que debe ser resuelto. Si a esto le sumamos el nivel educativo de la población, ello conlleva a que las actividades económicas desarrolladas en esta realidad sean limitadas.

La creación de micro empresas, no es algo establecido sino más bien es una iniciativa de los mismos probladores como medio de subsistencia más que como desarrollo, por lo tanto la hipotesis planteada queda demostrado que dista mucho de la realidad estudiada.

\section{MÉTODO Y TÉCNICAS UTILIZADAS}

Los métodos y técnicas empleadas para la elaboración del presente trabajo se expone a continuación:

\section{Métodos}

Los métodos utilizados variaron en relación a la formulación de la hipótesis y a la comprobación de la misma. Para la formulación de la hipótesis el método utilizado fue el deductivo, el que fue auxiliado por el método del marco lógico para formular la hipótesis y los objetivos de la investigación, diagramados en los árboles de problemas y objetivos que forman parte del anexo de este documento.

Para la comprobación de la hipótesis el método utilizado fue el inductivo, que contó con el auxilio de los métodos estadístico, de análisis y de síntesis.

La forma de empleo de los métodos citados se expone a continuación:

\section{Métodos y técnicas utilizadas para la formulación de la hipótesis}

Para la formulación de la hipótesis el método principal fue el deductivo, el cual permitió conocer aspectos generales del AA.HH. Puerto Pachacútec del distrito de Ventanilla, Región Callao. A este efecto, se utilizaron las técnicas que se especifican a continuación:

- Observación directa. Esta técnica se utilizó directamente en el asentamiento humano observandose los estilos de vida y a terceras personas que poseían relación directa e indirecta con los pobladores, dirigentes comunales, proveedores, entre otros.

- Investigación documental. Esta técnica se utilizó a efectos de determinar si existía algún otro estudio hecho anteriormente aplicado al AA.HH. Puerto Pachacútec, ver si se poseían documentos similares o relacionados con la problemática a investigar, a fin de no duplicar esfuerzos en cuanto al trabajo académico que desarrollada así como para obtener aportes y otros puntos de vista de otros investigadores sobre la temática citada. Luego de una búsqueda comprobamos que no había ningún estudio realizado antes.

- Entrevista. Una vez formada una idea general de la problemática se procedió a entrevistar a los pobladores a efectos de obtener información más precisa sobre la problemática de promocionar la actividad comercial. 
Teniendo una visión más clara sobre la problemática, con la utilización del método deductivo y a través de las técnicas anteriormente descritas, se procedió a la formulación de la hipótesis utilizandose el método del marco lógico, que permitió encontrar la variable dependiente e independiente de la hipótesis, además de definir el área de trabajo y el tiempo para desarrollar la investigación.

La hipótesis formulada de la forma indicada reza: "La promoción empresarial de la micro empresa como factor de desarrollo en el AA.HH. Puerto Pachacútec del distrito de Ventanilla, Región Callao".

El método del marco lógico nos permitió, entre otros aspectos, encontrar el objetivo general y el específico de la investigación, además de establecer la denominación del trabajo.

\section{Métodos y técnicas empleadas para la comprobación de la hipótesis}

Para la comprobación de la hipótesis el principal método utilizado fue el inductivo, con el que se obtener resultados específicos de la problemática identificada lo que sirvió para diseñar conclusiones y premisas generales a partir de tales resultados.

A este efecto se utilizaron las técnicas que se especifican a continuación:

- Entrevista. Previo a desarrollar la entrevista, se procedió al diseño de boletas de investigación con el propósito de comprobar las variables dependiente e independiente de la hipótesis previamente formulada. Las boletas, antes de ser aplicadas a población objetivo, tuvieron un proceso de prueba con la finalidad de hacer más efectivas las preguntas y propiciar que las respuestas proporcionaran la información requerida, después de ser aplicada.

- Determinación de la población a investigar. En atención a este tema, el grupo de investigación efectuó un muestreo estadístico que representara a la población a estudiar, la misma que estaba constituida por 100 personas que vivían en el asentamiento humano. Después de recabar la información contenida en las boletas, se procedió a tabularlas para cuyo efecto se utilizó el método estadístico y el método de análisis, que consistió en la interpretación de los datos tabulados, en valores absolutos y relativos, obtenidos después de la aplicación de las boletas de investigación, que poseyeron como objeto la comprobación de la hipótesis previamente formulada. Una vez interpretada la información se utilizó el método de síntesis para obtener las conclusiones y recomendaciones del presente trabajo de investigación. Esto sirvió además para hacer congruente la totalidad de la investigación con los resultados obtenidos producto del trabajo de campo efectuada.

\section{Técnicas}

Las técnicas empleadas tanto en la formulación como en la comprobación de la hipótesis se expusieron anteriormente, pero éstas variaron de acuerdo a la formulación de la hipótesis y a la comprobación de la misma; así las técnicas empleadas en la formulación fueron la observación directa y la entrevista a las personas relacionadas directamente con la problemática. Por otro lado, la comprobación de la hipótesis se realizó mediante la entrevista y el censo de negocios.

Como se puede advertir fácilmente, la entrevista estuvo presente en la etapa de la formulación de la hipótesis y en la etapa de la comprobación de la misma. La investigación documental estuvo presente en las dos etapas indicadas, en toda la investigación documental y especialmente para conformar el marco teórico.

\section{EXPOSICIÓN, SISTEMATIZACIÓN E INTERPRETACIÓN DE LOS RESULTADOS LOGRADOS}

La presente investigación busca conocer el potencial de desarrollo de las micro empresas del AA.HH. puerto Pachacútec para el establecimiento de un plan de aprovechamiento de la información lograda y, en base a esta, establecer proyectos que promueven el desarrollo socioeconómico.

\section{Trabajo de campo}

La recolección de la información se realizó mediante la aplicación de un cuestionario estandarizado para el muestreo de la población; para esto se plantean cinco resultados relacionados con: 
- Nivel de instrucción.

- Actividad económica desarrollada.

- Acceso a servicios públicos y tecnológicos.

- Acceso a medios y vías de transporte.

- Apoyo del gobierno central local y regional.

A continuación se describe en qué consiste cada uno de los resultados planteados:

1. Nivel de instrucción de la población como marco de desarrollo.

Este resultado busca conocer la situación actual del nivel de instrucción del poblador promedio. Se requiere saber qué posibilidades tiene un poblador de alcanzar su desarrollo económico basado en el nivel de instrucción.

2. Actividad económica desarrollada existente en la zona de ámbito de estudio.

Este resultado pretende desarrollar un diagnóstico de las distintas actividades que realiza los pobladores para tener una actividad que les permita generar ingresos, conocer y evaluar las potencialidades existentes para el futuro desarrollo de todo el asentamiento humano.

\section{Acceso a servicios públicos y tecnológicos}

Este resultado corresponde a los distintos tipos de acceso de la población hacia los servicios públicos y tecnológicos. La limitación existente de estos medios en los asentamientos humanos es preponderante como factor de desarrollo.

\section{Acceso a medios y vías de transporte}

Una de las condiciones necesarias para el desarrollo de la comunidad de Pachacutec es el contar con vías y medios de transporte. Los asentamientos humanos carecen de este tipo de acceso siendo este un limitante que los gobiernos deben implementar.

5. Apoyo del gobierno central, local y regional Las políticas establecidas por los gobiernos deben estar encaminadas al establecimiento de normas que asuman el desarrollo social y económico de la población. Los programas asistenciales complementados con campañas está encaminadas a la prestación de diversos servicios. Para tales propósitos se debe establecer la participación de la comunidad a fin de conocer las diversas necesidades existentes en los asentamientos humanos.
APLICACIÓN PRÁCTICA DE LOS RESULTADOS DE LA INVESTIGACIÓN

1. Nivel de instrucción de la población como marco de desarrollo

CUADRO N 1 NIVEL DE INSTRUCCIÓN PORCENTUAL

\begin{tabular}{|l|r|r|}
\hline & \multicolumn{1}{|c|}{ HOMBRES } & \multicolumn{1}{c|}{ MUJERES } \\
\hline PRIMARIA & $40 \%$ & $64 \%$ \\
\hline SECUNDARIA & $50 \%$ & $32 \%$ \\
\hline TÉCNICA & $10 \%$ & $4 \%$ \\
\hline UNIVERSITARIA & $0 \%$ & $0 \%$ \\
\hline \multicolumn{1}{|c|}{ TOTAL } & $100 \%$ & $100 \%$ \\
\hline
\end{tabular}

Gráfico N¹ Hombres

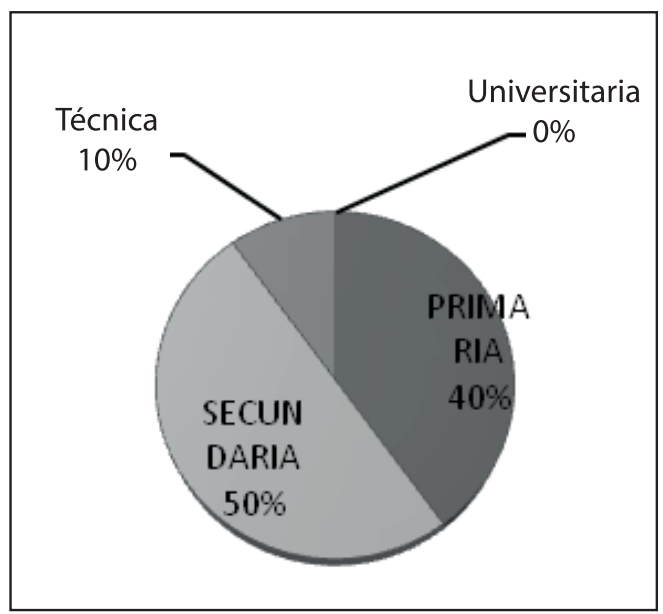

Gráfico $\mathrm{N}^{\circ} 2$ Mujeres

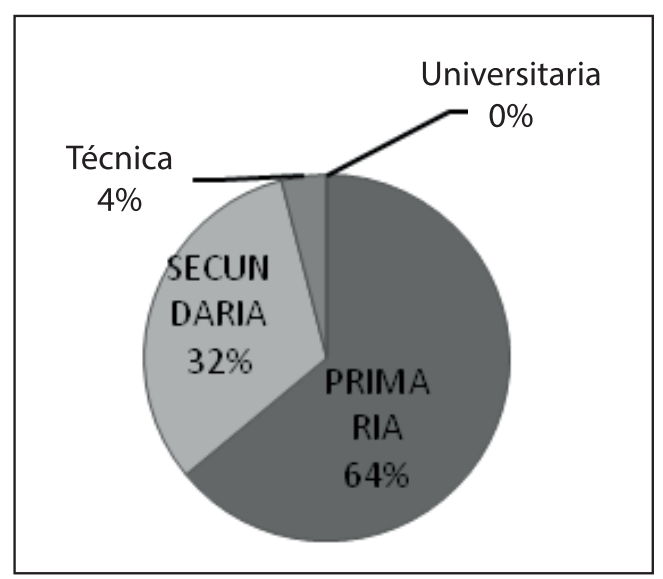

El nivel de instrucción es limitado lo que conlleva a que los pobladores que vean como opción el incursionar una actividad de micropempresa podrá tener ciertas limitaciones de poder emprender un negocio. Las personas en su mayoría son inmigrantes de las distintas provincias del Perú. Sobre este punto plantearemos unas propuestas en el presente trabajo. 
2. Actividad económica desarrollada existente en la zona de estudio

CUADRO N² ACTIVIDAD ECONÓMICA

\begin{tabular}{|c|c|c|}
\hline ACTIVIDADES & $\mathrm{N}^{0}$ & $\%$ \\
\hline COMERCIO & 8 & $42 \%$ \\
\hline SERVICIOS & 5 & $26 \%$ \\
\hline ARTESANIA & 2 & $11 \%$ \\
\hline EXTRACCIÓN & 4 & $21 \%$ \\
\hline TOTAL & 19 & $100 \%$ \\
\hline
\end{tabular}

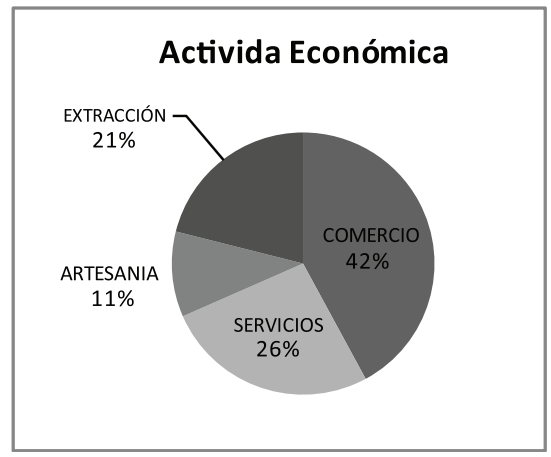

En el AA.HH. Puerto Pachacútec se desarrollan -en su mayoría- pequeños negocios de abarrotes o bodegas que cubren cierta necesidad de consumo en la zona. Sobre los servicios se ve la apertura de un colegio privado como parte del incipiente desarrollo. Así mismo, la cercanía al mar ha hecho que se aperturen algunos negocios de preparación de comida pero que son estacionales (sobre todo en los meses que comprende el verano). En menor grado se desarrollan actividades artesanía así como pesca artesanal (labor extractiva). Cabe destacar la futura construcción de un puerto artesanal cercano al asentamiento humano, lo que hará que las diversas actividades puedan ir incrementándose. Es importante precisar que el asentamiento humano está cerca de una congregación católica que desarrolla actividades educativas en el nivel escolar y técnico.

\section{Acceso a servicios públicos y tecnológicos}

CUADRO N ${ }^{\circ} 3$ ACCESO A SERVICIOS PÚBLICOS

\begin{tabular}{|c|c|c|}
\hline SERVICIO & TIPO & $\%$ DE USO \\
\hline AGUA & CISTERNA & $100 \%$ \\
\hline ALCANTARILLADO & NO & $0 \%$ \\
\hline LUZ & $\mathrm{SI}$ & $80 \%$ \\
\hline TELEFONÍA & RED MÓVIL & $70 \%$ \\
\hline CABLE & INALÁMBRICO & $10 \%$ \\
\hline INTERNET & INALÁMBRICO & $3 \%$ \\
\hline
\end{tabular}

Gráfico $N^{\circ} 4$ Servicios públicos

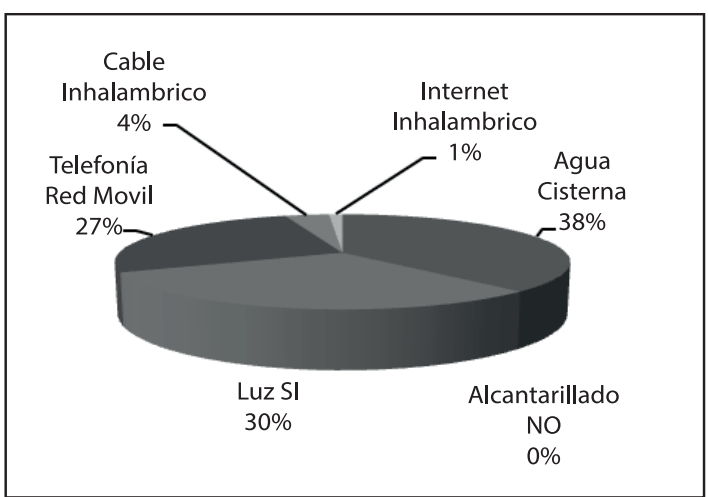

El acceso a los servicios públicos es muy diferenciado. Los básicos como agua son suministrados por cisternas, lo que hace que sea un servicio restringido. No existe el alcantarillado, sino el empleo de silos. En cuanto al servicio eléctrico, éste se ha implementado hace poco de una forma permanente a casi todo el asentamiento humano, estableciéndose ya una red eléctrica fija. Los servicios de cable son suministrados por una empresa privada bajo el sistema inalámbrico, pero solo a un grupo minoritario de viviendas; igual ocurre con el servicio de Internet.

\section{Acceso a medios y vias de transporte}

\begin{tabular}{|l|l|c|}
\hline \multicolumn{3}{|c|}{ CUADRO N ${ }^{\circ} 4$ ACCESO A MEDIOS Y VÍAS DE TRANSPORTE } \\
\hline SERVICIO & \multicolumn{1}{|c|}{ TIPO } & $\%$ \\
\hline TRANSPORTE PÚBLICO & LIVIANO & $85 \%$ \\
\hline TRANSPORTE PÚBLICO & PESADO & $10 \%$ \\
\hline TRANSPORTE PARTICULAR & MOTOCICLETAS - AUTOS & $5 \%$ \\
\hline \multicolumn{2}{|c|}{ TOTAL } & $100 \%$ \\
\hline Base: 100 personas - 50 Hombre 50 mujeres \\
\hline
\end{tabular}

Gráfico $N^{\circ} 5$ Acceso a medios de transportes

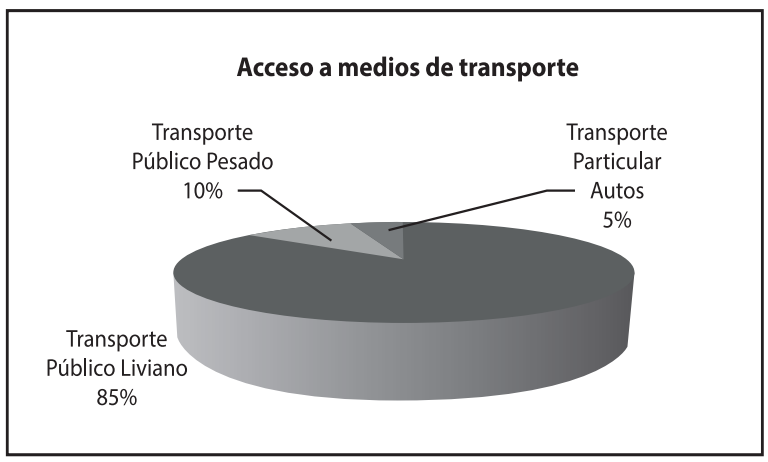

El acceso a medios de transporte en los asentamientos humanos tiene una característica en común: la proliferación de un transporte público de tipo liviano como las moto-taxis que son empleados por la gran mayoría de la población. El transporte en ómnibus es restringido porque 
solo tiene cobertura desde el centro de la capital hasta el inicio del asentamiento humano, lo que hace que el trasporte interno sea realizado por los moto-taxis. El transporte particular es minoritario y está representado por motocicletas y pocos autos que ingresan a la zona.

\section{Apoyo del gobierno central, regional y local}

CUADRO N 5 APOYO GUBERNAMENTAL

\begin{tabular}{|l|c|c|c|}
\hline \multirow{2}{*}{\multicolumn{1}{|c|}{ SERVICIO }} & \multicolumn{3}{c|}{ PERCEPCIÓN } \\
\cline { 2 - 4 } & APOYO & NO APOYO & TOTAL \\
\hline CONCEJO DISTRITAL & $30 \%$ & $70 \%$ & $100 \%$ \\
\hline CONCEJO PROVINCIAL & $10 \%$ & $90 \%$ & $100 \%$ \\
\hline CONCEJO REGIONAL & $8 \%$ & $92 \%$ & $100 \%$ \\
\hline Base: $\mathbf{1 0 0}$ personas - 50 hombre 50 mujeres \\
\hline
\end{tabular}

Gráfico Nº6 Apoyo gubernamental

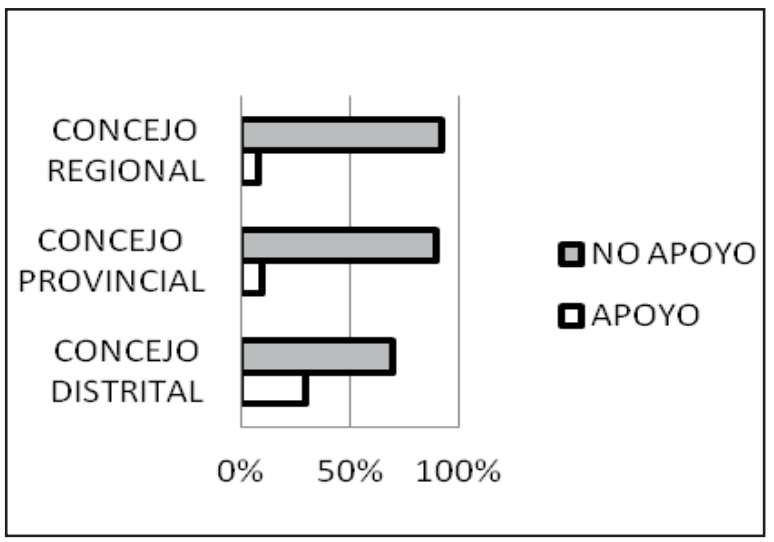

Respecto al apoyo gubernamental, la mayoría de entrevistados percibe que este es menor al que debería ser. Las campañas se circunscriben al aspecto de salud, básicamente. En cuanto a la promoción de desarrollo económico no es percibida como algo que los distintos gobiernos tengan en sus planes en el corto plazo. Una de las formas de promover la generación de micro empresas es hacer jornadas de capacitación y de sensibilización a fin de concientizar en la población el propósito del emprendimiento como factor de sostenibilidad en sus economías familiares.

De acuerdo a los resultados presentados, el responsable del presente proyecto elaboró un plan de charlas sobre capacitaciones a los pobladores que dieron a conocer su intención de emprender un negocio. Inicialmente estas capacitaciones buscaron captar la atención de los pobladores y posteriormente, les transmitieron conocimientos básicos en distintas áreas que serán de utilidad en los negocios.
Las áreas que formaron parte de las capacitaciones fueron:

- Documentación mercantil.

- Contabilidad.

- Marketing.

- Tributación.

En documentación mercantil, se les capacitó en la identificación de los distintos comprobantes de pago y su forma de registro; además de hacerles ver la importancia de la formalidad en cada transacción que puedan realizar.

En la segunda área de capacitación, contabilidad, se les informó acerca de las nociones básicas de las diversas herramientas contables para un mejor sistema de control de sus finanzas y de su inversión.

En la tercera área se les capacitó sobre estrategias de marketing en los negocios. Se les hizo saber que aun dentro de la limitación de recursos, todo negocio siempre debe tener formas de llegar a un mayor número de clientes.

En la última área, la de tributación, se les hizo conocer la legislatura existente sobre las micro empresas y la forma como ésta ayuda a que los costos laborales no sean iguales a los de una empresa ubicada en otro régimen. Asimismo se les inculcó sobre el poder tener una cultura de la formalidad y de cumplimiento.

Es importante precisar que estas charlas de capacitación se realizaron en varias fechas y con el apoyo de algunas personas del asentamiento. En este aspecto, finalmente, se requiere de una campaña permanente de capacitaciones a fin de poder lograr el objetivo de promocionar la micro empresa.

\section{CONCLUSIONES Y RECOMENDACIONES}

De acuerdo a los resultados elaborados y analizados, el presente proyecto pretende proponer medios y formas para el fomento de la micro empresa en el AA.HH. Puerto Pachacútec. La sensibilización en un sector de la población es uno de los objetivos plasmados en este proyecto, pero requiere de una permanente dedicación para que el emprendimiento mediante la micro empresa sea una opción para mejora y la calidad de vida. Es aquí donde las instituciones gubernamentales, especialmente; tienen que ir formulando sólidas políticas de desarrollo de este sector de la sociedad. 
En primer lugar el acceso a los servicios básicos es una prioridad, porque no hay desarrollo si no hay energía, agua y alcantarillado. No se puede promover el desarrollo de una comunidad sino cuenta con estos servicios básicos. El presente estudio debe servir a las autoridades para tomar en cuenta esta necesidad en el corto plazo. La realización de convenios con los pobladores para que apoyen mediante jornadas de trabajo sería algo viable, toda vez que este tipo de actividades, como aspecto cultural, está muy identificado con ellos. Debemos tener presente que los pocos negocios establecidos en la zona hacen notorios esfuerzos por tener acceso a estos servicios bajo distintas formas y medios, lo que genera un costo superior al que debería. Por ejemplo, en los asentamientos humanos se deben asumir un mayor costo del agua potable suministrada por camiones cisternas respecto a si tuvieran un servicio permanente de tuberías. En resumen, el desarrollo de las micro empresas en el AA.HH. Puerto Pachacutec tiene como factor crucial la implementación de los servicios públicos básicos.

Respecto a la promoción de las micro empresas desde el punto de vista de las capacitaciones, éstas podrán ser asumidas por las Direcciones correspondiente de los gobiernos locales y central para que puedan implementar diversos programas dirigidos al fomento de actividades económicas. Este aspecto debe ser planificado, contando con la participación de organizaciones educativas, universidad e institutos. Es importante establecer convenios de proyección social con las comunidades para que los pobladores reciban capacitaciones en áreas como contabilidad, elaboración de costos, publicidad entre algunas herramientas que ayudarán al futuro micro empresario a tener un mayor y mejor manejo de su unidad de producción.

Otro aspecto que debe ser tomando en cuenta es la formalización de los títulos de propiedad de cada poblador. Está demostrado que el poseer un título de propiedad será la base para que el futuro microempresario pueda tener acceso al crédito de fomento otorgado por instituciones financieras. Actualmente los programas de titulación son considerados por los políticos como uno de los instrumentos más efectivos para reducir la pobreza en los países en desarrollo. La idea, de acuerdo con investigadores como De Soto (2000), es proveer activos a los pobres y permitirles usarlos de manera irrestricta, de modo que puedan generar mayor riqueza. En ese contexto, diversos gobiernos han iniciado programas de titulación en países pobres. El Perú no es una excepción. Desde 1996, el gobierno peruano, a través del Organismo de Formación de la Propiedad Informal, inició un programa de titulación urbano de gran envergadura. Desde entonces, este programa es uno de los mayores esfuerzos gubernamentales dirigidos a la titulación de las zonas urbanas en el mundo, con más de 2 millones de títulos de propiedad registrados y más de 8 millones de habitantes beneficiados. De esta manera, el programa de titulación se ha constituido en una de las principales apuestas de los gobiernos para luchar contra la pobreza urbana y fomentar la movilidad social.

Finalmente con este estudio se pretende fomentar el desarrollo de un gran asentamiento humano como es Puerto Pachacútec y buscar generar una cultura de emprendedores, con la mejora continua de su calidad de vida y también de su desarrollo económico.

\section{LITERATURA CITADA}

Calva-Mercado, A. (1998). "La microempresa grande y la grande micro”.[En línea]. Fecha de consulta: 17/04/2013 Artículo completo en: http://www.acus.com.mx/. México.

Campaña Nuñez, Aldo. (s.f.). “¿Qué sucede con las micro empresas en el Perú?”. En: Universidad del Pacífico. (s.f.). Saberes Compartidos. [En línea]. Fecha de consulta: 19/04/2013. Disponible en: <http://www.saberescompartidos.pe/ciencias-sociales-y-politicas/ que-sucede-con-las-microempresas-en-elperu.html > .

Hernando de Soto, Enrique Ghersi , Mario GhibeIlini, Instituto libertad y Democracia (1987) "El otro Sendero" Editorial Oveja Negra, Lima -Perú.

Matos Barzola, Alan Emilio. (2008). "Tributación de MYPEs". En: Matos Barzola, Alan Emilio. (2008). Blog Tributación Perú. [En línea]. Fecha de consulta: 06/05/2013. Disponible en: <http://alanemiliomatosbarzola.wordpress.com/2008/09/05/mypes-microempresas-y-pequenas-empresas-peru/ $>$.

Micro empresa (s.f.). En: Wikipedia. [En línea]. Fecha de consulta: 08/05/2013.Disponible en: < http://es.wikipedia.org/wiki/Microempresa $>$.

Organización Internacional del Trabajo (OIT). (2006). Panorama Laboral 2006, América Latina y el Caribe. OIT/ Oficina Regional para América Latina y el Caribe, Lima.

Ruiz, C. (2004). Dimensión territorial del desarrollo económico de México. UNAM, Facultad de Economía, México. 